\title{
Análisis estadístico de la base de datos climatológica del parámetro humedad relativa de Honduras
}

César Joel Martínez Romero

\section{RESUMEN}

Honduras es un país con un territorio predominantemente montañoso, de clima cálido y húmedo, cuya economía se basa en gran parte en la producción agrícola, rubro cuyo rendimiento puede verse influenciado por diferentes factores climáticos, tales como la humedad relativa.

La humedad relativa es un parámetro meteorológico que en Honduras es monitoreado por aproximadamente un $17 \%$ del total de estaciones meteorológicas con que se cuenta el país. Desafortunadamente, esta cobertura no es tan extensa como se esperaría; además, gran parte de la información que se brinda presenta vacíos importantes. En consecuencia, si se quiere trabajar de manera más eficiente no solo en el campo de la agricultura, sino también en las áreas de ingeniería, generación de energía, etc., es necesario crear una plataforma de datos históricos de humedad relativa que sea confiable.

La presente investigación tiene como objetivo la recopilación ordenada y sistemática de la mayor cantidad de información histórica posible sobre el parámetro de humedad relativa, de modo que esta pueda ser analizada y completada de manera confiable. Para el desarrollo de la investigación se ha recolectado información de alrededor de 50 estaciones, a cuyos datos se les realizó una serie de análisis estadísticos y de continuidad. Estas estaciones se rellenaron mediante la utilización de un programa creado por la Cooperación Española, en 2002, en conjunto con la SERNA. El producto final de este estudio es una base de datos de humedad relativa continua, homogénea y aleatoria de este parámetro en Honduras.

Palabras clave: humedad relativa, serie histórica, análisis estadístico, base de datos.

\footnotetext{
${ }^{1}$ Profesor universitario beneficiario de una beca especial de la DICYP. Maestría de Metodologías de Investigación Económica y Social.
} 


\section{ABSTRACT}

Honduras is a highly mountainous country, with a warm and humid weather, itseconomy is strongly based in agriculture and food production. The performance of this economic field could be influenced by different climatic factors such as Relative Humidity.

Relative humidity is a meteorological parameter which is measured in Honduras by $17 \%$ of the total of meteorological agencies. Unfortunately, there is no enough information as we would expect; moreover, most of the data that we do have is not $100 \%$ complete. Thus, if we want to work in a more efficient way, not only in agriculture, but also in the engineering field or the energy field, it is necessary to create a historical relative humidity database.

This research aim is to collect the most relative humidity data we can, so it can be studied and statistically analyzed. In this paper, we have acquired information from about 50 meteorological agencies. This data has been tested, completed and revised; making sure that we count with a continuous and homogeneous database. To complete the information provided by the different agencies, we have used a software that was developed in 2002 by the Spanish Cooperation Agency in Honduras. With the completion of this research, we can supply with a complete, homogeneous and reliable database in Honduras.

Key words: relative humidity, historical series, statistical analysis, database. 


\section{INTRODUCCIÓN}

El clima hondureño es tropical, de elevadas temperaturas y mucho más húmedo en la vertiente antillana que en la pacífica. En las montañas el clima es más templado, mientras que en la costa norte las temperaturas son muy altas. Pero, este concepto debe ser más específico para cada una de los sectores del territorio nacional, por lo que es necesario tener una base de datos de humedad relativa en el país.

La humedad relativa es la humedad que contiene una masa de aire con relación a la máxima humedad absoluta que podría admitirse sin producir condensación conservando las mismas condiciones de temperatura y presión atmosférica. Es un factor climático que puede modificar el rendimiento final de los cultivos, ya que la humedad alta favorece a la transmisión de plagas, enfermedades y abortos florales y la humedad baja podría secar las plantas.

Cada especie tiene una humedad ambiental idónea para vegetar en perfectas condiciones, por eso es una variable climatológica que necesita tener una base de datos confiable, razón por la cual esta investigación estará orientada a definir esa base de datos, recolectando la información de las diferentes instituciones (públicas y privadas), luego procesarla (analizarla) a fin de tener datos confiables y saber cómo varía la humedad relativa en las diferentes zonas de nuestro territorio.

Honduras cuenta con alrededor de 300 estaciones meteorológicas, de las cuales la mayoría solo toma datos de precipitación y únicamente un $17 \%$ toma datos de humedad relativa; además, de estas estaciones muchas presentan problemas con los datos recolectados, ya que existen muchos meses, incluso años, en los que estas estaciones no presentan datos, lo que se convierte en uno de los mayores problemas para analizar los datos estadísticamente, por eso para poder tener una base de datos confiable se debe tener una base de datos aleatoria, homogénea y continua.

\section{OBJETIVOS}

\section{General}

Analizar estadísticamente la base de datos climatológica del parámetro humedad relativa, a fin de determinar si la serie es homogénea, aleatoria y continua, para que 
pueda ser utilizada en el balance hídrico en el país y estudios relacionados con el tema.

\section{Específicos}

1. Recopilar la base de datos del parámetro humedad relativa de todas las instituciones (SERNA, SMN, ENEE, SANAA, FHIA, UNAH, sector privado) a fin de contar con todas las series históricas disponibles en el país; así como todos los estudios relacionados que se hayan hecho.

2. Hacer un análisis estadístico de las series históricas obtenidas, a fin de determinar si las series son homogéneas, aleatorias y continuas.

\section{METODOLOGÍA}

El tipo de investigación realizada es descriptiva, cuyo fin será conocer de manera más profunda cómo es la humedad relativa en el territorio nacional. La metodología aplicada se resume en los siguientes pasos:

1. Recolectar la información de las diferentes instituciones encargadas de medir las variables climatológicas (SERNA, SMN, ENEE, SANAA, FHIA, UNAH, sector privado).

2. Estandarizar la información a un solo formato en común.

3. Análisis estadístico de la calidad y consistencia de los datos.

4. Elaboración de la base de datos.

Recolección de la información

Los datos meteorológicos en el país son recolectados por instituciones públicas (SERNA, ENEE, SANAA, FHIA, SMN) y privadas (Zamorano), también se consideraron en el análisis 2 estaciones de El Salvador (La Unión y Planes de Montecristo). La mayoría de los datos recolectados en el país son de precipitación. A la fecha, alrededor de 300 estaciones toman datos de este parámetro y de estas solo alrededor del $17 \%$ toman datos de humedad relativa.

Para esta investigación se recolectaron todos los datos posibles de las instituciones antes mencionadas, pero cabe señalar que estas instituciones 
manejan sus datos con un formato en el que tienen todos los parámetros en conjunto, por lo que al pedir los datos de humedad relativa también se incluían datos de los demás parámetros.

La recolección de los datos se hizo con el apoyo del Instituto Hondureño de Ciencias de la Tierra (IHCIT). Se visitaron las instituciones encargadas de la medición de estos datos, información que es de carácter exclusivo, por lo que utilizarla para esta investigación resultó en algún momento difícil debido al accesode los mismos.

\section{Estandarizar la información a un solo formato en común}

Una vez recolectada la información se procedió a estandarizarla a un formato con el cual se pudieran hacer los análisis correspondientes y así comenzar a elaborar la base de datos. Para realizar esta etapa, que cada institución maneja con su propio formato para la recolección de los datos (algunas manejan el registro de los datos diarios, otras mensuales y otras diariamente), fue necesario, previo al análisis, tener todos los datos de las estaciones en un formato común que permitiera poder analizar los datos de manera adecuada, para ello se elaboró una plantilla para todas las estaciones del país.

La mayoría de las instituciones recolectan sus datos y mantienen sus registros en año calendario, pero para esta investigación los datos se colocaron en formato de año hidrológico (Rascon, 2005). Este se establece para intentar reflejar adecuadamente el comportamiento de las precipitaciones sobre una determinada cuenca hidrográfica.

El comienzo del año hidrológico puede variar entre una región y otra, incluso dentro de un mismo país. Para Honduras se considera que comienza el 1 de mayo y finaliza el 30 de abril. Se decidió colocar la humedad relativa en formato de año hidrológico para coincidir con el análisis de la precipitación que se realiza en el país y así poder hacer análisis comunes entre estos 2 parámetros; también para el cálculo de la evapotranspiración potencial (ETP) del país, que es un factor importante para realizar el balance hídrico de Honduras.

Además en la plantilla se colocó otra información como el nombre de la estación, institución que recolecta los datos, un código que corresponda al que maneja cada institución, departamento y la cuenca a la que pertenece la estación, datos para ubicar la estación geográficamente como coordenadas UTM y geográficas y elevación en metros sobre el nivel del mar (msnm). Toda esta información ayuda a 
encontrar una relación común entre una estación y otra para hacer correlaciones al momento de hacer el relleno de datos faltantes.

\section{Análisis estadístico de la calidad y consistencia de los datos}

Una vez obtenidos todos los datos necesarios y estandarizada la información a un formato común, se debe determinar la calidad y consistencia de los datos recolectados, lo cual se realiza mediante pruebas estadísticas; estas validan si un dato es correcto o si está fuera de los parámetros estadísticos establecidos.

Además de pruebas estadísticas, es necesario hacer una revisión comparativa entre datos de estaciones con iguales características, ya que un dato válido estadísticamente no necesariamente es correcto; un ejemplo es la humedad relativa, debido a que es un parámetro climatológico que varía muy poco de una región a otra, por eso la similitud de los datos recolectados entre una estación y otra deben cumplir lo anteriormente descrito. Un caso de esto se dio entre la estación Marale y la estación Las Lajas, pertenecientes a la cuenca del Ulúa, las que presentan datos para mayo de 1976 de $76.3 \%$ y $78.3 \%$, respectivamente, lo que demuestra que el dato recolectado es correcto. Del mismo modo, los datos dentro de la misma estación deben presentar cierta similitud y tendencia a través de los años.

Los métodos estadísticos para analizar datos climáticos

Los procesos climáticos presentan irregularidades que son fuente de incertidumbre, ante lo cual la estadística permite describir los datos, cuantificar la incertidumbre y realizar inferencias.

La aplicación de métodos estadísticos adecuados debe ayudar a la comprensión de los fenómenos físicos subyacentes y la valoración de la hipótesis. El análisis estadístico de los datos puede sugerir la existencia (o no) de relaciones entre variables y la descripción de los datos observados puede ayudar a la predicción.

\section{Análisis de continuidad}

De las estaciones que toman datos de humedad relativa en el país, muchas no cuentan con la cantidad de datos necesarios para poder ser analizadas estadísticamente, por lo que una de las primeras cosas realizadas en esta investigación fue determinar la continuidad de la serie histórica de cada una de las 
estaciones meteorológicas. Para ello se ingresaron los datos en un formato requerido para el $\mathrm{CHAC}^{2}$ y se realizó un gráfico de continuidad de las estaciones (ver gráfico 1). En este gráfico se observa la falta de datos que presentan las estaciones en algunos periodos. Para este análisis se descartaron aquellas estaciones que contaban con muy pocos datos en su serie histórica (5 años o menos),ya que el programa CHAC descarta estaciones con muy pocos datos.

Gráfico 1. Datos de continuidad de las diferentes estaciones de Honduras

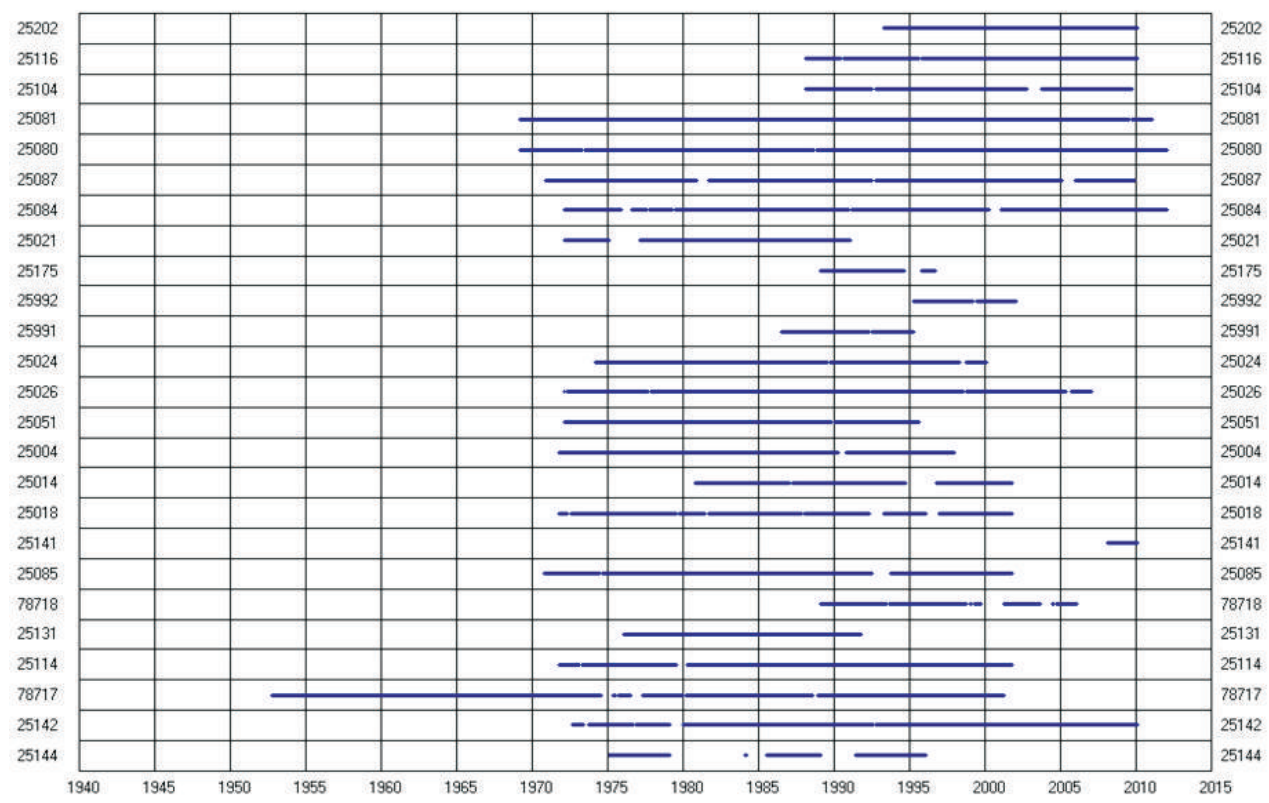

Consistencia de los datos

Los datos de cada una de las estaciones se colocaron en plantillas en las cuales se determinaron parámetros estadísticos como media, desviación estándar, curtosis, sesgo, valores máximos, valores mínimos. Para determinar la consistencia de los datos se revisaron los datos para ver si sus valores estaban dentro del rango permisible para un cierto nivel de significación.

Se determinaron los datos raros (datos que no presentan un comportamiento normal en la serie histórica), marcando aquellos que eran mayores o menores que la media \pm 3.5 veces la desviación estándar y de esta manera establecer un límite en la

${ }^{2}$ CHAC: programa utilizado para el análisis y relleno de datos faltantes. 
variabilidad de los datos recolectados. Además, también se identificaron aquellos datos que presentaban una humedad relativa de $0 \%$ y $100 \%$ en condiciones que existen en la realidad, pero que no es cierto que se dan en todas las zonas, especialmente en nuestro país en donde el clima es muy poco variable entre una región y otra; no sucede así en otros países en los que se pueden observar condiciones climáticas extremas.

Basándose en esto se identificaron los datos con anomalías para tenerlos presentes a la hora de hacer el relleno. Cada dato fuera del rango estadístico establecido se marcó en color rojo y se verificó si correspondía a las condiciones de la zona. Inicialmente, aunque un dato se considere como raro, este no debe descartarse, pues debe verificarse comparándolo con los datos de otras estaciones; si al hacer esta comparación el dato salía disparado, se podía tomar como descartado (ver gráfico 2).

Gráfico 2. Análisis estadístico, identificación de datos raros

\begin{tabular}{|c|c|c|c|c|c|c|c|c|c|c|c|c|}
\hline B & C & D & E & F & G & H & I & J & K & L & M & N \\
\hline $2001-02$ & 67 & 65 & 67 & 71 & 76 & 80 & 77 & 76 & 73 & 67 & 60 & 52 \\
\hline $2002-03$ & 61 & 75 & 76 & 73 & 74 & 78 & 76 & 72 & 74 & 67 & 62 & 61 \\
\hline $2003-04$ & 62 & 76 & 76 & 77 & 76 & 79 & 82 & 81 & 74 & 66 & 64 & 60 \\
\hline $2004-05$ & 69 & 71 & 70 & 63 & 69 & 73 & 77 & 75 & 68 & 58 & 59 & 55 \\
\hline $2005-06$ & 69 & 76 & 77 & 74 & 73 & 70 & $(62$ & 84 & 89 & 85 & 82 & $(85$ \\
\hline $2006-07$ & 86 & 84 & 80 & 77 & 76 & 82 & 83 & 85 & 80 & 73 & 72 & 72 \\
\hline $2007-08$ & & & & & & & & & & & & \\
\hline $2008-09$ & & & & & & & & & & & & \\
\hline $2009-10$ & & & & & & & & & & & & \\
\hline $2010-11$ & & & & & & & & & & & & \\
\hline $2011-12$ & & & & & & & & & & & & \\
\hline & & & & & & & & & & & & \\
\hline II & 20 & 19 & 21 & 21 & 21 & 20 & 20 & 19 & 21 & 21 & 21 & 21 \\
\hline Máxim0 & 86.0 & 84.0 & 80.0 & 81.0 & 83.0 & 83.0 & 83.0 & 90.0 & 89.0 & 85.0 & 82.0 & 85.0 \\
\hline Mínim0 & 52.0 & 64.0 & 67.0 & 63.0 & 68.0 & 70.0 & 62.0 & 72.0 & 66.0 & 56.0 & 54.0 & 51.0 \\
\hline MEDIA & & 72.2 & 73.0 & 72.5 & 75.1 & 77.7 & 77.6 & 77.5 & 73.2 & 67.1 & 62.4 & 59.5 \\
\hline DESVEST & 6.7 & 4.7 & 3.5 & 4.2 & 4.0 & 3.2 & 4.2 & 4.9 & 4.9 & 5.8 & 6.2 & 7.2 \\
\hline CURTOSIS & 4.981 & 1.211 & -0.839 & 0.158 & -0.345 & 0.389 & 10.757 & 0.903 & 4.441 & 4.204 & 4.267 & 7.756 \\
\hline SESGO & 1.354 & 0.324 & 0.109 & -0.263 & 0.035 & -0.347 & -2.798 & 1.120 & 1.663 & 1.032 & 1.769 & 2.494 \\
\hline Espacio.Blanco & 48 & 49 & 47 & 47 & 47 & 48 & 48 & 49 & 47 & 47 & 47 & 47 \\
\hline & & & & & & & & & & & & \\
\hline CV & \#iDIV/0! & 0.065 & 0.048 & 0.058 & 0.053 & 0.042 & 0.054 & 0.063 & 0.067 & 0.086 & 0.099 & 0.121 \\
\hline 3.5 Desv Tip & 23.3 & 16.4 & 12.2 & 14.7 & 14.0 & 11.4 & 14.6 & 17.0 & 17.3 & 20.2 & 21.5 & 25.3 \\
\hline T max & 23.3 & 88.5 & 85.2 & 87.1 & 89.1 & 89.0 & 92.2 & 94.6 & 90.5 & 87.4 & 84.0 & 84.8 \\
\hline T min & -23.3 & 55.8 & 60.8 & 57.8 & 61.2 & 66.3 & 63.0 & 60.5 & 55.9 & 46.9 & 40.9 & 34.2 \\
\hline
\end{tabular}

La existencia de un dato raro se puede deber a diferentes causas, tales como daño en el equipo que mide los datos, una mala lectura por parte de la persona encargada de recolectar la información, no tomar lectura en un determinado día debido a que generalmente la persona encargada de tomar la lectura de los datos trabaja con 
horario de oficina, lo cual hace que algunos días no se tome lectura o en condiciones ideales que para esa fecha la variabilidad del clima haya sido tal que provocara condiciones climáticas fuera de lo normal, dando como resultado datos disparados. Esto se puede comprobar con el simple hecho de comparar estaciones cercanas, las cuales deben presentar la misma variabilidad climática. Este análisis se hizo con detalle en la recolección de los datos usados para el presente artículo.

Se determinaron gráficos del comportamiento histórico de las estaciones que miden el parámetro humedad relativa, para observar si tenían un comportamiento igual en todos los años con datos; también como una ayuda para observar datos que no tuvieran un comportamiento similar y la continuidad de los datos (ver gráfico 3 ).

Grafico 3. Serie histórica de humedad relativa

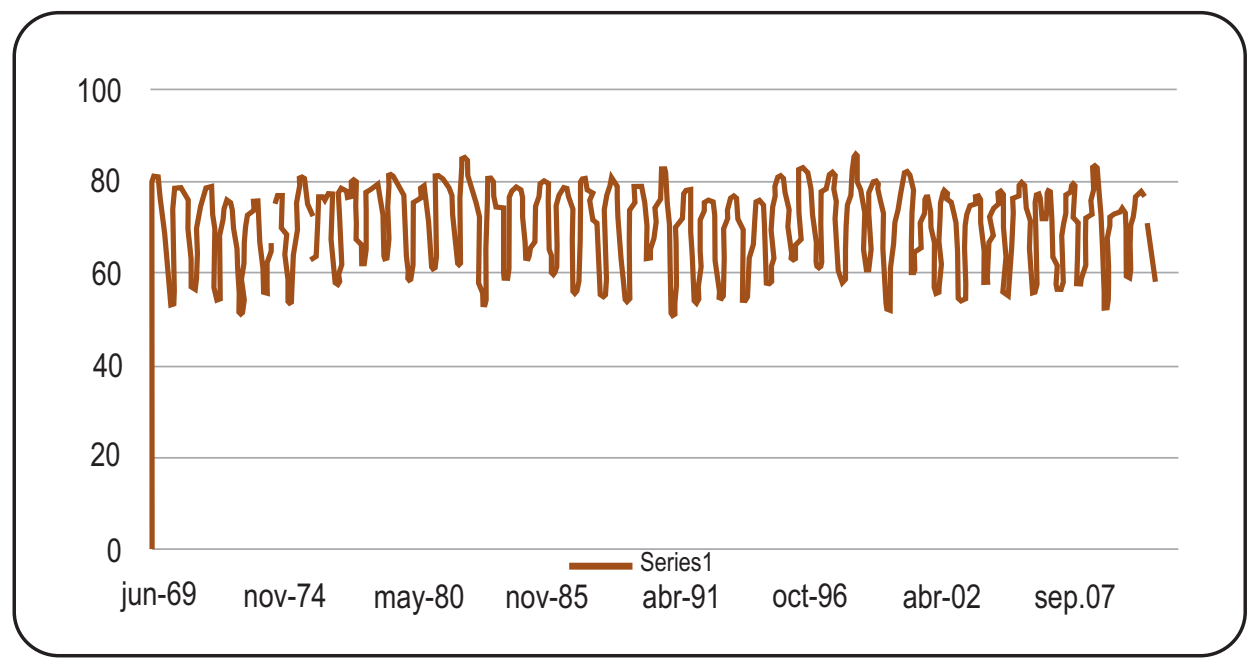

Criterios para completar datos

Para completar los datos se consideraron varios criterios, tales como serie histórica común, relación entre una estación y otra, tendencia histórica y ubicación geográfica.

1. Serie histórica común: para este relleno de datos se tomó una serie histórica de 30 años, 1975-2005. Esta fue la serie ingresada en el CHAC, los datos de las estaciones fueron rellenados solo para estos años. Estaciones que tenían serie histórica de antes de 1975 o después de 2005 no se rellenaron sus datos vacíos. 
Se tomó esta serie porque los datos recolectados de la mayoría de las estaciones estaban entre estos años, muy pocas estaciones tienen datos antes de esta fecha y no se pudo recolectar información reciente de algunas estaciones.

2. Relación entre una estación y otra: se buscó ver si la humedad relativa se podía agrupar de acuerdo a la altura de la zona propiamente, comparando la tendencia observada para cada una de las estaciones, encontrando que ciertas estaciones presentan esta característica, principalmente en las zonas costeras en donde a menor altura hay mayor humedad relativa; pero, otras estaciones no presentaron muy claro este comportamiento, lo que indica que la humedad no depende propiamente de la altura, sino también de otros factores adicionales como la temperatura, precipitación, velocidad del viento, etc. (ver gráfico 4)

Gráfico 4. Relación altura-humedad relativa, \% anual

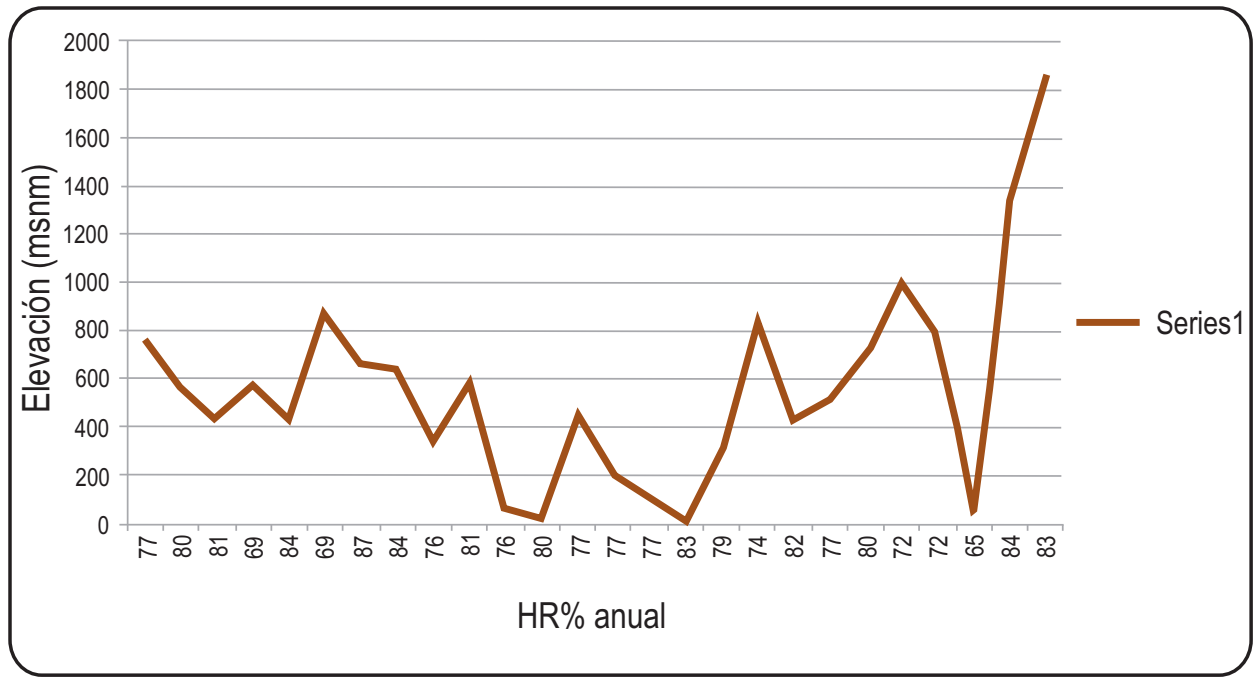

3. Tendencia histórica: la mayoría de las estaciones del país presentan la misma tendencia a través del tiempo. La humedad relativa varía muy poco durante los meses del año y generalmente todas presentaron las mismas tendencias. Las estaciones que presentaron una tendencia un poco diferente se verificaron sus datos (ver gráfico 5). 
Grafico 5. Variación de la humedad relativa para las estaciones analizadas

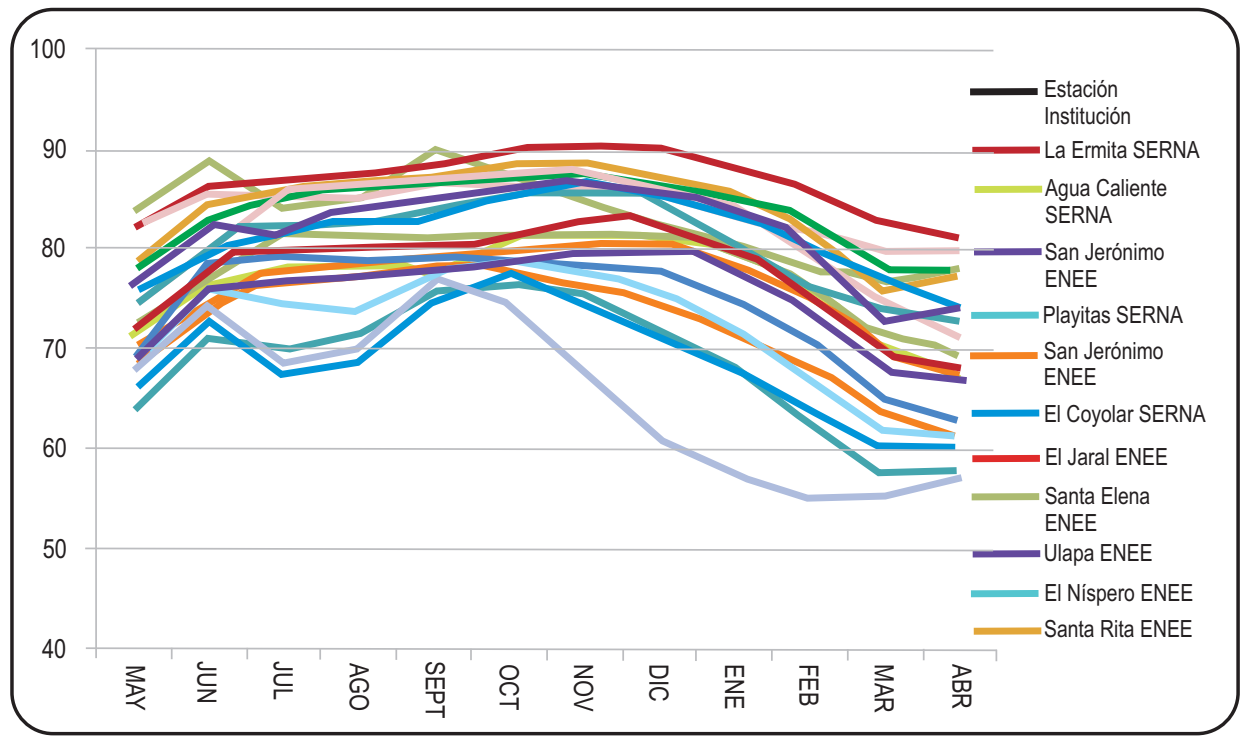

4. Ubicación geográfica: la humedad relativa en Honduras y en otros países presenta diferencias significativas entre la zonas costeras y la zona central; además muestra variaciones entre una costa y otra, ya que en la costa Atlántica, pese a que las temperaturas son elevadas, el clima es más húmedo; mientras que en la zona sur el clima es más seco. Para el relleno de datos se agruparon las estaciones por cuencas hidrográficas, entre las que tenemos: Choluteca, Chamelecón, Patuca, Ulúa, Nacaome, Cangrejal-Aguán, Leán, Segovia, Sico y Cuyamel; incluso cuencas que son muy grandes, como la del Ulúa, se separaron en estaciones que están en la cuenca alta y cuenca baja, para poder tener resultados más acertados. Se realizaron 31 grupos de estaciones agrupadas de acuerdo a su cercanía y considerando que presentaban las mismas condiciones climáticas.

\section{Completado de datos}

Existen muchas maneras para realizar el completado de datos, por ejemplo completar datos con la media, esto se puede realizar cuando el coeficiente de variación es menor o igual a 0.15 . El método de las dobles acumulaciones es una técnica para valorar la consistencia de una serie temporal en función de otra serie de referencia; para su aplicación se toman las dos series de datos durante el periodo 
común de registro, comparándose en un diagrama x-y sus series acumuladas. Si la relación entre las dos series se ha mantenido estable, es decir, a los incrementos de una corresponden los proporcionales en la de referencia, la representación mostrará una tendencia lineal; en caso contrario, la presencia de quiebros y saltos indica cambios en la relación entre las series de datos y por tanto posibles errores.

Las primeras aproximaciones realizadas en este artículo se hicieron con el método de dobles acumulaciones, pero se observó que debido a la falta de datos de muchas estaciones las correlaciones presentaban quiebres considerables, lo que provocaba que los datos no se ajustaran a la correlación necesaria para el análisis. En esta investigación, para completar las lagunas en las series de datos, se utilizó el programa CHAC, el cual se describe a continuación.

Descripción del programa de cálculo hidrometeorológico de aportaciones y crecidas (CHAC)

El programa utilizado para el relleno de los datos se llama cálculo hidrometeorológico de aportaciones y crecidas(CHAC), desarrollado por el Centro de Estudios Hidrográficos del CEDEX, de la Cooperación Española. Se realizó a partir de un modelo de regresión bivariado con estacionarización previa mensual de las series de datos (CORMUL). Para ello, generaron los ficheros LEMA, agrupando las estaciones por cuenca hidrográfica.

La secuencia de cálculos para la realización de la correlación múltiple queda especificada en el fichero de resultados intermedios. En primer lugar aparecen las matrices correspondientes a los datos mensuales de las estaciones seleccionadas para el completado en el periodo de estudio escogido. Las estaciones seleccionadas son aquellas que tienen más de cinco datos en cada uno de los doce meses en ese periodo seleccionado.

La elección de la pareja de estaciones que va a ser utilizada para el completado de cada una de las lagunas de una estación dada, se basa en considerar un umbral mínimo del coeficiente de correlación múltiple ponderado, según la expresión de la matriz de priorización. 
Fases operativas del completado de datos

1. Estacionarización de las series de datos de humedad relativa: $t_{i, j}=\frac{X_{i, j}-X_{j}}{S_{j}} t_{i, j}$ : es una serie estacionaria en media y varianza, sin tendencias estacionales

Siendo: $\quad \bar{X}_{j}=\frac{1}{N} \sum_{j=1}^{N} X_{i, j} \overline{S_{j}}=\sqrt{\frac{1}{N} \sum_{j=1}^{N}\left(X_{i, j}-\bar{X}_{j}\right)^{2}}$

Donde:

$$
\begin{array}{cl}
X_{i, j} & \text { :humedad relativa para el mes j del año I } \\
X_{j} & \text { :media del mes j } \\
\frac{N}{S_{j}} & \text { :número de años de la serie } \\
& \text { :desviación típica del mes j }
\end{array}
$$

2. Establecimiento de la ecuación de regresión. Expresa los valores ti,j $j^{3}$ de una estación como función de los de otra pareja de estaciones (ti, $j^{1}$ ti,j):

$$
t_{i, j}{ }^{3}=\bar{t}_{i, j}{ }^{3}+a_{1}\left(t_{i, j}{ }^{1}-\bar{t}_{i, j}{ }^{1}\right)+a_{2}\left(t_{i, j}{ }^{2}-\bar{t}_{i, j}{ }^{2}\right)+\varepsilon_{i, j}
$$

Donde:

1,2,3 : corresponden al número de orden de estación

$a_{1} y a_{2} \quad$ : son los coeficientes de regresión parcial, función de los coeficientes de correlación simple $r_{1,3} r_{2,3} r_{1,2}$

$\varepsilon_{i, j} \quad: \quad$ : es un ruido independiente de distribución normal con media

$\bar{t}_{i, j}{ }^{1}, \bar{t}_{i, j}{ }^{2} y \bar{t}_{i, j}{ }^{3}: \begin{gathered}\text { valor medio de la serie estacionarizada de cada estación, su } \\ \text { valor será } 0\end{gathered}$

3. Proceso de completado según la matriz de priorización que permite la elección de la pareja de estaciones (i,j) con que se completa una tercera (k) en función de su correlación y el número de datos comunes entre las tres estaciones:

Siendo:

$$
P_{i, j}{ }^{\mathrm{k}}=R_{i, j}{ }^{\mathrm{k}}\left(\frac{N_{i, j}{ }^{\mathrm{k}}}{N_{\text {ciclo }}}\right)^{a}
$$


$P_{i, j}{ }^{\mathrm{k}}:$ coeficiente de correlación múltiple ponderado por el número de meses con datos comunes, coeficiente de priorización

$N_{i, j}{ }^{\mathrm{k}}$ :número de meses con datos comunes entre las series correspondientes a las estaciones i, jyk

$N_{\text {ciclo }}$ :número de meses total del ciclo de completado

a :exponente de priorización

4. Desestacionarización de las series de datos:

$$
X_{i, j}=\bar{X}_{j}+\bar{S}_{j} * t_{i, j}
$$

El programa genera un fichero LEMA con los datos de cada una de las estaciones ya rellenado en formato bloc de notas.

\section{Elaboración de la base de datos}

Una vez obtenidos los datos rellenados se procedió a colocarlos en plantillas en las cuales se presentan los datos para un periodo desde 1975 a 2005, los resultados presentados en esta base de datos son de 49 estaciones a nivel nacional.

\section{RESULTADOS}

1. Tener una base de datos con formato homogéneo de 49 estaciones de humedad relativa en el país, la cual servirá como una plataforma para estudios relacionados con el tema (ver cuadro 1).

Cuadro 1. Resumen de datos promedio de humedad relativa para 49 estaciones a nivel nacional

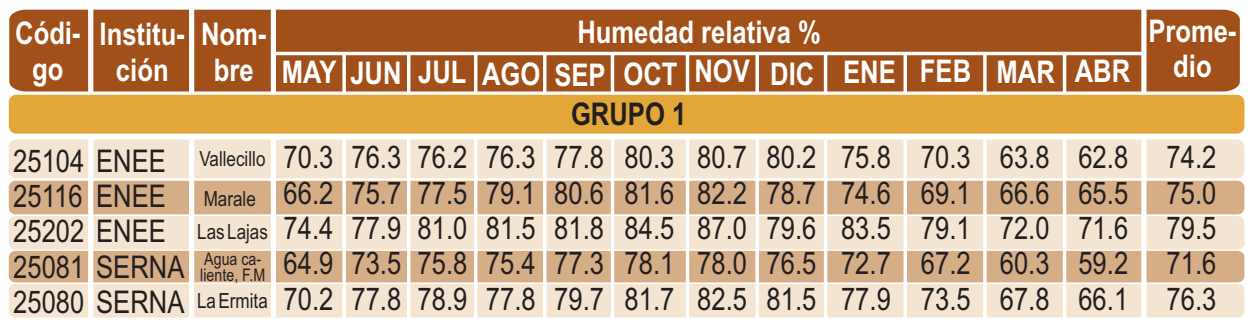




\begin{tabular}{|c|c|c|c|c|c|c|c|c|c|c|c|c|c|c|c|}
\hline \multirow{2}{*}{$\begin{array}{c}\text { Códl- } \\
\text { go }\end{array}$} & \multirow{2}{*}{$\begin{array}{c}\text { Institu- } \\
\text { ción }\end{array}$} & \multirow{2}{*}{$\begin{array}{c}\text { Nom- } \\
\text { bre }\end{array}$} & \multicolumn{12}{|c|}{ Humedad relativa $\%$} & \multirow{2}{*}{$\begin{array}{c}\text { Prome- } \\
\text { dio }\end{array}$} \\
\hline & & & MAY & JUN & JUL & AGO & SEP & OCT & NOV & DIC & ENE & FEB & MAR & ABR & \\
\hline \multicolumn{16}{|c|}{ GRUPO 2} \\
\hline 25087 & ENEE & $\begin{array}{l}\text { San } \\
\text { Jerónimo }\end{array}$ & 77.0 & 82.7 & 82.6 & 84.2 & 85.8 & 87.2 & 87.4 & 86.6 & 84.0 & 79.8 & 74.2 & 74.9 & 81.8 \\
\hline \multicolumn{16}{|c|}{ GRUPO 3} \\
\hline 5021 & SERNA & $\begin{array}{l}\text { Siguatia- } \\
\text { peque }\end{array}$ & 73.1 & 77.5 & 77.7 & 79.0 & 80.8 & 83.6 & 83.9 & 82.7 & 81.3 & 76.2 & 71.8 & 68.5 & 78.0 \\
\hline 084 & & Playitas & 63.4 & 70.8 & 70.0 & 70.8 & 75.9 & 76.8 & 76.2 & 72.8 & 69.3 & 62.9 & 58.3 & 58.1 & 68.8 \\
\hline \multicolumn{16}{|c|}{ GRUPO 4} \\
\hline $0<1$ & SERNA & $\begin{array}{l}\text { Santa } \\
\text { Calara }\end{array}$ & 64.3 & 73.2 & 74.2 & 73.2 & 74.7 & 75.6 & 77.2 & 77.9 & 74.6 & 68.8 & 61.8 & 60.1 & 71.3 \\
\hline 5024 & SERNA & Las Flores & 69.7 & 75.3 & 72.9 & 68.2 & 77.6 & 78.9 & 77.1 & 74.9 & 70.4 & 67.5 & 63.9 & 64.2 & 71.7 \\
\hline 026 & SERNA & El Coyolar & 66.8 & 73.2 & 67.8 & 69.8 & 76.0 & 77.8 & 76.8 & 73.2 & 69.1 & 64.7 & 61.4 & 61.3 & 69.8 \\
\hline \multicolumn{16}{|c|}{ GRUPO 5} \\
\hline 004 & SERNA & La Gloria & 75.9 & 81.6 & 82.1 & 81.3 & 82.9 & 83.5 & 83.3 & 82.0 & 82.7 & 80.3 & 76.9 & 75.0 & 80.6 \\
\hline \multicolumn{16}{|c|}{ GRUPO 6} \\
\hline 08 & ENEE & Santa Elena & 79.1 & 83.9 & 85.9 & 87.2 & 87.2 & 87.2 & 87.8 & 87.6 & 86.5 & 84.0 & 80.2 & 80.3 & 84.8 \\
\hline & & El Cajön & 75.8 & 84.0 & 86.4 & 86.9 & 86.6 & 88.5 & 89.5 & 88.7 & 86.7 & 82.6 & 76.6 & 74.0 & 83.5 \\
\hline \multicolumn{16}{|c|}{$\begin{array}{ll}\text { GRUPO } 7 \\
\end{array}$} \\
\hline 12 & SERNA & Sensenti & 71.7 & 80.2 & 78.6 & 79.0 & 81.3 & 81.1 & 80.3 & 78.8 & 75.3 & 72.8 & 68.3 & 67.7 & 76.3 \\
\hline & & Ocotepeque & 68.1 & 75.9 & 73.7 & 74.1 & 78.6 & 76.6 & 72.8 & 68.6 & 65.6 & 62.5 & 60.2 & 1.6 & 70.3 \\
\hline \multicolumn{16}{|c|}{ GRUPO 8} \\
\hline 2 & ENEE & Ulapa & 69.2 & 76.3 & 78.2 & 79.9 & 81.3 & 82.5 & 82.2 & 81.4 & 78.3 & 74.1 & 68.2 & 66.8 & 76.7 \\
\hline & & $\begin{array}{l}\text { Sta. Rosa } \\
\text { de Copan }\end{array}$ & 75.7 & 81.0 & 82.4 & 82.9 & 83.7 & 84.6 & 86.0 & 85.6 & 83.0 & 79.7 & 73.6 & 72.9 & 81.0 \\
\hline \multicolumn{16}{|c|}{ GRUPO 9} \\
\hline 2514 & ENEE & EI Nispero & 74.7 & 81.7 & 82.3 & 83.0 & 84.1 & 85.1 & 86.3 & 85.5 & 83.7 & 79.0 & 75.1 & 73.9 & 81.2 \\
\hline \multicolumn{16}{|c|}{ GRUPO 10} \\
\hline & FHIA & Guaruma 1 & 77.2 & 79.1 & 80.3 & 80.4 & 80.1 & 82.5 & 83.5 & 83.8 & 81.7 & 79.9 & 77.3 & 76.4 & 80.2 \\
\hline & SMN & Catacamas & 65.1 & 73.8 & 77.1 & 77.0 & 78.3 & 78.7 & 79.0 & 77.0 & 73.6 & 67.4 & 63.1 & 61.0 & 72.6 \\
\hline & FHIA & Calán & 80.4 & 81.1 & 82.0 & 83.0 & 82.4 & 84.3 & 85.3 & 85.2 & 84.5 & 82.7 & 80.0 & 80.0 & 82.6 \\
\hline & ENEE & Santa Rita & 69.1 & 75.2 & 77.9 & 77.7 & 78.0 & 79.5 & 81.2 & 81.7 & 79.0 & 76.5 & 70.8 & 70.0 & 76.5 \\
\hline 2 & SERNA & Morazán & 75.9 & 81.0 & 82.3 & 82.1 & 82.1 & 84.3 & 84.3 & 83.8 & 82.7 & 81.2 & 77.9 & 76.3 & 81.2 \\
\hline & SMN & La Mesa & 74.0 & 77.1 & 79.3 & 79.8 & 78.9 & 82.2 & 85.1 & 85.3 & 84.1 & 81.3 & 76.8 & 74.5 & 80.1 \\
\hline \multicolumn{16}{|c|}{ GRUPO 12} \\
\hline & & Quimistán & 70.1 & 76.0 & 77.8 & 77.6 & 78.2 & 80.3 & 81.4 & 81.2 & 79.1 & 76.0 & 71.0 & 68.7 & 76.5 \\
\hline & & La Entrada & 71.0 & 77.6 & & 80.4 & $\begin{array}{r}81.0 \\
\text { GR }\end{array}$ & $\begin{array}{l}81.8 \\
\text { UPO } 1\end{array}$ & 82.1 & 82.1 & 79.7 & 76.5 & 70.2 & 68.4 & 77.6 \\
\hline
\end{tabular}

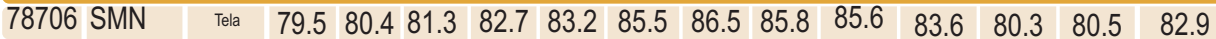

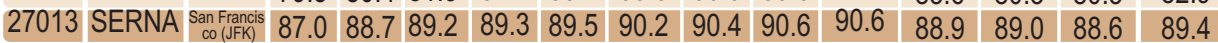

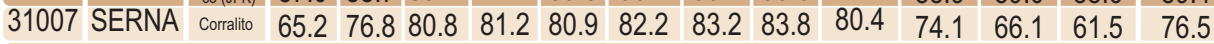
GRUPO 16

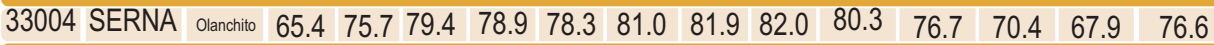
GRUPO 18

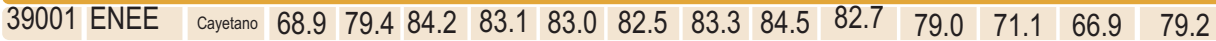
GRUPO 19

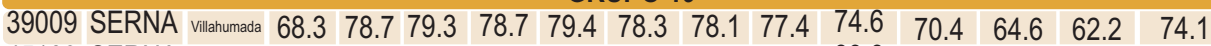

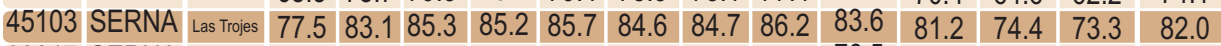

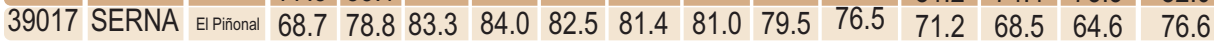
GRUPO 20

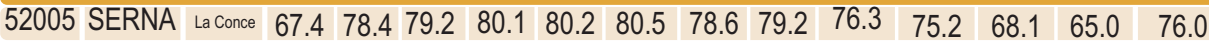

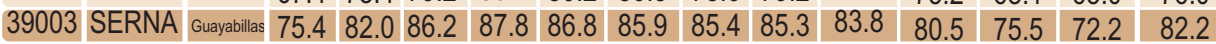
GRUPO 21

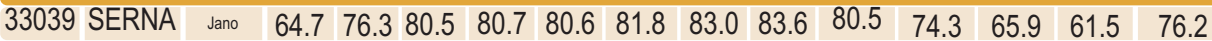
GRUPO 23

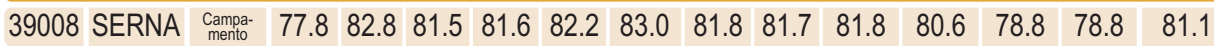




\begin{tabular}{|c|c|c|c|c|c|c|c|c|c|c|c|c|c|}
\hline \multirow{2}{*}{$\begin{array}{c}\text { Códi- } \\
\text { go }\end{array}$} & \multirow{2}{*}{$\begin{array}{l}\text { Institu- } \\
\text { ción }\end{array}$} & \multirow{2}{*}{$\begin{array}{c}\text { Nom- } \\
\text { bre }\end{array}$} & \multicolumn{10}{|c|}{ Humedad relativa $\%$} & \multirow{2}{*}{$\begin{array}{c}\text { Prome- } \\
\text { dio }\end{array}$} \\
\hline & & & \begin{tabular}{|l|l|} 
MAY JUN \\
\end{tabular} & JUL $A G O$ & SEP & OCT & NOV & DIC & ENE & FEB & MAR & ABR & \\
\hline \multicolumn{14}{|c|}{ GRUPO 24} \\
\hline 3720 & SMN & $\begin{array}{l}\text { Tegucici } \\
\text { galpa }\end{array}$ & 68.475 .0 & 73.673 .3 & 76.9 & 78.7 & 78.6 & 75.6 & 72.1 & 67.4 & 62.5 & 62.7 & 72.1 \\
\hline \multicolumn{14}{|c|}{ GRUPO 26} \\
\hline 001 & SERNA & -a Venta & 63.671 .6 & 72.372 .1 & 75.0 & 77.2 & 76.9 & 76.7 & 72.5 & 66.3 & 61.0 & 57.8 & 70.3 \\
\hline $0 /$ & & $\begin{array}{l}\text { Ingenio } \\
\text { Porvenir }\end{array}$ & $74.8 \quad 83.2$ & $85.4 \quad 82.2$ & 84.4 & 84.6 & 83.4 & 82.3 & 76.9 & 76.0 & 70.6 & 70.1 & 79.6 \\
\hline \multicolumn{14}{|c|}{ GRUPO 27} \\
\hline 00 & ERNA & $\begin{array}{c}\text { ElZamo- } \\
\text { rano }\end{array}$ & 66.9 & 76.7 & 78.8 & 77.8 & 77.0 & 75.0 & 71.9 & 68.8 & 64.6 & 62.0 & 72.7 \\
\hline \multicolumn{14}{|c|}{ GRUPO 30} \\
\hline 002 & SERNA & $\begin{array}{c}\text { Monteli- } \\
\text { bano }\end{array}$ & 76.0 & 73.0 & 82.2 & 81.1 & 72.9 & 67.3 & 60.4 & 58.7 & 59.9 & 65.1 & 71.1 \\
\hline 0023 & SERNA & La Lujosa & 83.184 .5 & 83.183 .1 & 85.4 & 84.4 & 82.4 & 81.9 & 83.6 & 80.8 & 82.8 & 82.8 & 74.6 \\
\hline 8724 & SMN & Choluteca & 67.372 .2 & 68.269 .2 & 75.0 & 73.5 & 68.2 & 63.9 & 60.5 & 57.5 & 56.7 & 58.8 & 65.7 \\
\hline 001 & SERNA & Pespire & 67.273 .8 & $65.6 \quad 68.5$ & 77.0 & $\mid 77.1$ & 68.2 & 61.7 & 55.3 & 50.1 & 51.2 & 53.6 & 64.4 \\
\hline \multicolumn{14}{|c|}{ GRUPO 31} \\
\hline & RNA & Marcala & 82.4 & 85.285 .1 & 86.4 & 86.3 & 85.9 & 85.8 & 83.6 & 81.7 & 79.5 & 79.7 & 83.9 \\
\hline 6999 & ENEE & $\begin{array}{c}\text { La Espe- } \\
\text { ranza }\end{array}$ & $\begin{array}{ll}77.8 & 83.1\end{array}$ & \begin{tabular}{ll|}
81.7 & 82.3
\end{tabular} & 83.7 & 84.5 & 84.7 & 83.9 & 80.8 & 75.7 & 72.6 & 74.4 & 80.4 \\
\hline
\end{tabular}

2. Se determinó el número de estaciones que han tomado datos de humedad relativa a través de los años para observar cómo han ido aumentando las estaciones que toman datos de este parámetro (ver gráfico 6).

Grafico 6. Número de estaciones que tomaban humedad relativa

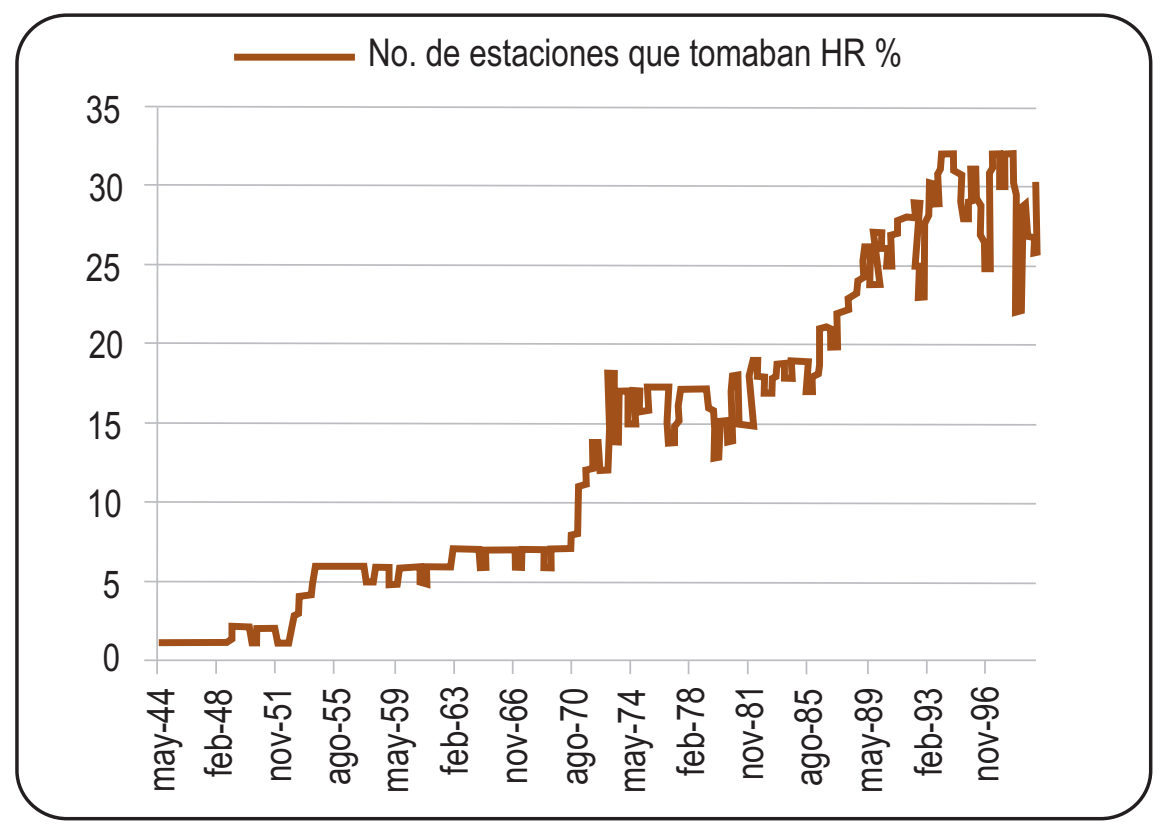




\section{CONCLUSIONES}

1. Al realizar esta base de datos se deja un precedente importante para su posterior uso, considerando que para tomar datos debemos tenerlos ordenados de manera consistente, además se debe tener una continuidad y homogeneidad entre los datos existentes.

2. Las estaciones analizadas fueron 49 , pero esta cantidad es muy baja considerando la necesidad que existe de tener información de esta variable climatológica y de otras para hacer estudios hidrometeorológicos.

3. Se ha visto un crecimiento en el número de estaciones que toman datos de humedad relativa en el país con el paso de los años, pero esta cantidad aún no es suficiente, por lo que las instituciones encargadas de esta labor (SERNA, SMN, ENEE, SANAA, FHIA, UNAH, sector privado) deben unir esfuerzos para continuar ampliando la cobertura de los datos y además existen zonas del país de las que no se tiene ningún registro de estos datos, lo que genera una deficiencia en la representatividad de los datos a nivel nacional.

\section{AGRADECIMIENTOS}

A José Castejón, Ronald Castellón, Fernando Borjas, Kelly Almendares y Marcela Garay, quienes colaboraron en la elaboración de la base de datos. Asimismo, gracias a Tania Peña, MaxAyala, Anahí Vega y Lenin Henríquez por sus consejos y apoyo logístico durante todo este proceso.

\section{BIBLIOGRAFÍA}

CEDEX. (2012). CHAC. Cálculo hidrometeorológico de aportaciones y crecidas. Madrid.

Centro de Estudios Hidrográficos del CEDEX. (2002). Balance hídrico de Honduras. Tegucigalpa.

Henríquez Fierro, E. y Zepeda Gonzales, M. I. (2004). Elaboración de un artículo científico de investigación. Ciencia y Enfermería, 1,6. 
Maderey Rascon, Laura Elena. (2005). Principios de hidrogeografía. Estudio del ciclo hidrológico. México: Universidad Autónoma de México.

Rascon, M. y Jiménez Román, L. E. (2005). Principios de hidrogeografía. Estudio del ciclo hidrológico. México D.F.: Serie de Textos Universitarios. 